NORDITA-93/30 N

SUNY-NTG-93-7

\title{
FROM KAON-NUCLEAR INTERACTIONS TO KAON CONDENSATION
}

\author{
G.E. Brown ${ }^{a}$ Æ, Chang-Hwan Lee \\ a Department of Physics, State University of New York \\ Stony Brook, N.Y. 11794, U.S.A. \\ ${ }^{b}$ Department of Physics, Seoul National University \\ Seoul 151-742, Korea \\ ${ }^{c}$ Service de Physique Théorique, C.E. Saclay \\ F-91191 Gif-sur-Yvette, France \\ ${ }^{d}$ NORDITA, Blegdamsvej 17, DK-2100 Copenhagen Ø, Denmark
}

\begin{abstract}
An effective chiral Lagrangian in heavy-fermion formalism whose parameters are constrained by kaon-nucleon and kaon-nuclear interactions next to the leading order in chiral expansion is used to describe kaon condensation in dense "neutron star" matter. The critical density is found to be robust with respect to the parameters of the chiral Lagrangian and comes out to be $\rho_{c} \sim(3-4) \rho_{0}$. Once kaon condensation sets in, the system is no longer composed of neutron matter but of nuclear matter. Possible consequences on stellar collapse with the formation of compact "nuclear stars" or light-mass black holes are pointed out.
\end{abstract}

\footnotetext{
${ }^{*}$ Supported in part by the Department of Energy under Grant No. DE-FG02-88ER40388

${ }^{\dagger}$ Supported by the Korea Science and Engineering Foundation through the CTP of Seoul National University
} 


\section{Introduction}

Kaon condensation in dense nuclear and neutron matter predicted first by Kaplan and Nelson [1] has recently been reformulated in chiral perturbation theory (ChPT) to the leading chiral order by Politzer and Wise [2] and by Brown et al [3] with results indicating dramatic consequences in stellar collapse [3, 4, 5] and neutron star cooling [3, 4]. In all these publications, the condensation process is mainly driven by the attraction gained as the $K N$ sigma term $\Sigma_{K N}$ associated with explicit breaking of chiral symmetry

$$
\Sigma_{K N} \approx \frac{1}{2}\left(\bar{m}+m_{s}\right)\langle N|(\bar{u} u+\bar{s} s)| N\rangle
$$

is "rotated out" by nuclear density, where $\bar{m}=\left(m_{u}+m_{d}\right) / 2$ is the mean up- and downquark mass, $m_{s}$ the s-quark mass and $u, s$ are the relevant quark fields. The $K N$ sigma term (1) which depends on the s-quark content of the proton could in principle be determined from experiment just as the $\pi N$ sigma term could be determined from $\pi N$ scattering. Unfortunately neither is the strangeness content of the proton known nor are $K N$ scattering data accurate enough to determine the quantity $\Sigma_{K N}$. If we assume as an extreme case that $\langle\bar{s} s\rangle_{N} \equiv\langle N|\bar{s} s| N\rangle \approx 0$, then we obtain $\Sigma_{K N} \sim 1.3 m_{\pi}$ at the tree order with the chiral Lagrangian we write down below. If on the other hand we take $\langle\bar{s} s\rangle_{N} /\langle(\bar{u} u+\bar{d} d)\rangle_{N} \approx 0.2$ which some of current hadrons models predict, we get $\Sigma_{K N} \sim 3.7 m_{\pi}$. In the literature, this range of magnitude of the sigma term has led to what appears to be a fairly robust prediction for kaon condensation at a matter density three or four times nuclear matter density $\rho_{0}\left(\approx \frac{1}{2} m_{\pi}^{3}\right)$. We shall consider this to be the range of values to keep in mind in what follows, although generally favoring the value of

$$
\Sigma_{K N} \sim 6 \Sigma_{\pi N} \sim 2 m_{\pi}
$$

using the empirical value of the $\pi N$ sigma term. The choice of this value will be justified later.

The problem with this prediction based on the scalar attraction due to (11) is that by itself such an attraction is not compatible with available data on kaon-nucleon and kaonnuclear interactions at low energy. As pointed out by several authors [6], there seems to be no serious need for a remnant of the scalar attraction in S-wave $K^{ \pm} N$ and $K^{ \pm}$-nuclear scattering amplitudes with the bulk of the data adequately accounted for by standard $\rho$ and $\omega$-meson-exchange mechanisms [7].

The purpose of this paper is to show that kaon condensation does still occur at the same low density, $\left(\sim(3-4) \rho_{0}\right)$, with an effective chiral Lagrangian that is compatible with low-energy kaon-nuclear interactions. The critical density could be lowered even further if 
one invokes the previously proposed in-medium scaling (which we shall call "BR scaling") in the chiral Lagrangians [9]. We shall do this using heavy-fermion chiral perturbation expansion [10] to next to the leading order. We shall consider a chiral Lagrangian consisting of the octet baryons $B=B^{a} T^{a}$ and the octet pseudoscalar mesons $\pi=\pi^{a} T^{a}$ in heavyfermion formalism (HFF) [10], first in free space and then in medium. We will focus on Swave kaon-baryon interactions, so the decuplet baryons which we expect to be unimportant for this partial wave will not be included in our consideration. Although vector mesons do not figure explicitly in our effective Lagrangian, their chiral symmetry and scaling properties in nuclear medium will be invoked as in ref.[9] to infer the scaling behavior of the relevant term in the Lagrangian.

\section{Chiral Perturbation Theory}

In arranging terms in a consistent chiral expansion for mesons and baryons, it proves to be convenient to use the Weinberg counting rule [11] according to which an amplitude involving $E_{N}$ number of external nucleon lines and $E_{K}$ number of external kaon lines can be characterized by $Q^{\nu}$ in the amplitude where $Q$ is the characteristic small momentum scale involved in the process and

$$
\nu=2+2 L-\frac{1}{2} E_{N}+\sum_{i}\left(d_{i}+\frac{1}{2} n_{i}-2\right)
$$

where $L$ is the number of loops, the sum over $i$ goes over all vertices, $d_{i}$ the number of derivatives that act on the $i$ th vertex and $n_{i}$ the number of nucleon lines attached to the $i$ th vertex. In the absence of external fields, chiral symmetry constrains\}

$$
P_{i} \equiv d_{i}+\frac{1}{2} n_{i}-2 \geq 0 .
$$

Applied to $K N$ scattering, we see that the leading term in this counting is given by $L=0$ and $P_{i}=0$ which is satisfied by a vertex with $d_{i}=1$ and $n_{i}=2$, so the amplitude has the index $\nu=1$. At the next order, we can have $L=0$ and one $P_{i}=1$ vertex with an index $\nu=2$. We note that no loops contribute to this order. One-loop terms contribute at the next order, say, $\nu=3$ together with tree graphs involving one vertex with $d_{i}=2$. In this paper, we will limit ourselves up to $\nu=2$ for kaon-nucleon scattering and hence no loops need be calculated. Applied to kaon-nuclear scattering and to kaon condensation, this procedure effectively takes into account density-dependent one-loop contributions consistently

\footnotetext{
${ }^{3}$ In the presence of slowly varying electroweak fields, the constraint is $P_{i} \geq-1$. This is important in nuclear exchange currents [12].
} 
with chiral symmetry as discussed in [2]. This chiral counting is embodied in the chiral Lagrangian first written down by Jenkins and Manohar [10] which we shall use:

$$
\mathcal{L}=\mathcal{L}_{0}+\mathcal{L}^{\prime}
$$

where

$$
\begin{aligned}
\mathcal{L}_{0} & =\frac{f^{2}}{4} \operatorname{Tr} \partial_{\mu} U \partial^{\mu} U^{\dagger}+r \operatorname{Tr} M(U+\text { h.c. }-2) \\
& +\operatorname{Tr} \bar{B} i v \cdot D B+2 D \operatorname{Tr} \bar{B} S^{\mu}\left\{A_{\mu}, B\right\}+2 F \operatorname{Tr} \bar{B}\left[A_{\mu}, B\right], \\
\mathcal{L}^{\prime} & =a_{1} \operatorname{Tr} \bar{B}(\xi M \xi+\text { h.c. }) B+a_{2} \operatorname{Tr} \bar{B} B(\xi M \xi+\text { h.c. })+a_{3} \operatorname{Tr} \bar{B} B \operatorname{Tr}(M U+\text { h.c. }) \\
& +c_{1} \operatorname{Tr} \bar{B} D^{2} B+c_{2} \operatorname{Tr} \bar{B}(v \cdot D)^{2} B \\
& +d_{1} \operatorname{Tr} \bar{B} A^{2} B+d_{2} \operatorname{Tr} \bar{B}(v \cdot A)^{2} B+d_{3} \operatorname{Tr} \bar{B} B A^{2}+d_{4} \operatorname{Tr} \bar{B} B(v \cdot A)^{2} \\
& +d_{5} \operatorname{Tr} \bar{B} B \operatorname{Tr} A^{2}+d_{6} \operatorname{Tr} \bar{B} B \operatorname{Tr}(v \cdot A)^{2}+d_{7} \operatorname{Tr} \bar{B} A_{\mu} \operatorname{Tr} A^{\mu} B \\
& +d_{8} \operatorname{Tr} \bar{B}(v \cdot A) \operatorname{Tr}(v \cdot A) B+d_{9} \operatorname{Tr} \bar{B} A_{\mu} B A^{\mu}+d_{10} \operatorname{Tr} \bar{B}(v \cdot A) B(v \cdot A) \\
& +f_{1} \operatorname{Tr} \bar{B}(v \cdot D)(S \cdot A) B+f_{2} \operatorname{Tr} \bar{B}(S \cdot D)(v \cdot A) B+f_{3} \operatorname{Tr} \bar{B}\left[S^{\alpha}, S^{\beta}\right] A_{\alpha} A_{\beta} B \\
& +\cdots
\end{aligned}
$$

where $v^{\mu}$ is the four-velocity of the heavy baryon (with $v^{2}=1$ ) and

$$
\begin{aligned}
D^{\mu} B & =\partial^{\mu} B+\left[V^{\mu}, B\right], \\
V^{\mu} & =\frac{1}{2}\left(\xi \partial^{\mu} \xi^{\dagger}+\xi^{\dagger} \partial^{\mu} \xi\right), \quad A^{\mu}=\frac{1}{2 i}\left(\xi \partial^{\mu} \xi^{\dagger}-\xi^{\dagger} \partial^{\mu} \xi\right)
\end{aligned}
$$

with $\xi^{2}=U$. Here $S^{\mu}$ is the spin operator $S^{\mu}=\frac{1}{4} \gamma_{5}\left[\not, \gamma^{\mu}\right]$ constrained to $v \cdot S=0, a_{i}$, $c_{i}, d_{i}$ and $f_{i}$ are parameters to be fixed later. Except for some minor differences, we use the notations of Jenkins and Manohar 10] where the advantage of using this Lagrangian for chiral perturbation theory is clearly pointed out. This Lagrangian was recently shown to be suited for describing chiral properties of nuclear systems by Park et al[12] where one can also find detailed discussions on how to systematically compute higher chiral-order terms using this Lagrangian.

We now illustrate the counting rule discussed above in terms of the Lagrangian (5). The leading order $K N$ (say $K^{+} N$ ) scattering is described by $\mathcal{L}_{0}$. Specifically the $\nu=1$ S-wave $K N$ amplitude is given by $i \operatorname{Tr} \bar{B}\left[V^{0}, B\right]$ which can be written explicitly as

$$
\mathcal{L}_{\nu=1}=\frac{-i}{8 f^{2}}\left(3\left(\bar{N} \gamma^{0} N\right) \bar{K} \overleftrightarrow{\partial}_{t} K+\left(\bar{N} \vec{\tau} \gamma^{0} N\right) \cdot \bar{K} \vec{\tau} \overleftrightarrow{\partial}_{t} K\right)
$$

with $N^{T}=(p \quad n), K^{T}=\left(K^{+} K^{0}\right)$ and $\bar{K} \stackrel{\leftrightarrow}{\partial}_{t} K \equiv \bar{K}_{\vec{\partial}} \vec{t}_{t} K-\bar{K} \overleftarrow{\partial}_{t} K$. For $\bar{K} N$ scattering, due to G-parity, the isoscalar term changes sign. In terms of an effective Lagrangian that 
contains vector mesons such as hidden gauge symmetry Lagrangian of ref.[13], the first term of (10) can be identified as the $\omega$ exchange and the second term as the $\rho$ exchange between the kaon and the nucleon. Thus we can think of the leading-order contribution as vector-dominated. This is in agreement with the standard phenomenological mesonexchange picture [7]. Now the next chiral order amplitude comes from $\mathcal{L}^{\prime}$ (7). For S-wave $K N$ scattering, the terms with the coefficients $c_{i}$ and $f_{i}$ do not contribute, so the relevant part of the Lagrangian simplifies (for S-wave) to

$$
\begin{aligned}
\mathcal{L}_{\nu=2}= & \frac{\Sigma_{K N}}{f^{2}}(\bar{N} N) \bar{K} K+\frac{C}{f^{2}}(\bar{N} \vec{\tau} N) \cdot(\bar{K} \vec{\tau} K) \\
& +\frac{\tilde{D}}{f^{2}}(\bar{N} N) \partial_{t} \bar{K} \partial_{t} K+\frac{\tilde{D}^{\prime}}{f^{2}}(\bar{N} \vec{\tau} N) \cdot\left(\partial_{t} \bar{K} \vec{\tau} \partial_{t} K\right)
\end{aligned}
$$

where

$$
\begin{aligned}
\Sigma_{K N} & =-\left(\frac{1}{2} a_{1}+a_{2}+2 a_{3}\right) m_{s}, \\
C & =-\frac{a_{1} m_{s}}{2}, \\
\tilde{D} & =\frac{1}{4}\left(d_{1}+d_{2}+d_{7}+d_{8}\right)+\frac{1}{2}\left(d_{3}+d_{4}\right)+\left(d_{5}+d_{6}\right), \\
\tilde{D}^{\prime} & =\frac{1}{4}\left(d_{1}+d_{2}+d_{7}+d_{8}\right) .
\end{aligned}
$$

As stated, the Lagrangian (10,11) at tree order gives the leading amplitude with $\nu=1$ and the next-to-leading amplitude with $\nu=2$. Loops with (6) give $\nu \geq 3$ and loops involving (7) even higher. Thus follows the statement that to order $\nu=2$ there are no loop contributions. This makes the calculation simpler than in $\pi \pi$ or $K K$ interactions where the next-to-leading order corrections involve both higher derivative counter terms and one-loop terms. This counting result in HFF is manifestly simpler than the relativistic formulation of ChPT [14] where numerous counter terms - none of which can be determined from other processes intervene.

In the strategy of chiral perturbation theory, the constants that appear in (10) and (11) are to be determined from experiments. As mentioned above, the $K N$ sigma term $\Sigma_{K N}$ could, in principle, be determined from low-energy $K N$ scattering data or from the expression (1) once we know the strangeness content of the nucleon $\langle N|\bar{s} s| N\rangle$. At the moment the sigma term is not known. We will take the value quoted above for our discussion. As for the coefficients $\tilde{D}$ and $\tilde{D}^{\prime}$ we anticipate an important contribution from the $1 / m_{B}$ correction due to baryon-antibaryon pair terms. These " $1 / m_{B}$ " corrections can be readily evaluated in the formalism (see Park et al [12]). The argument is sketched in the Appendix. 
Here we simply give the results

$$
\begin{aligned}
& \tilde{D}_{\frac{1}{m}} \approx-\frac{1}{48}\left[(D+3 F)^{2}+9(D-F)^{2}\right] / m_{B} \approx-0.12 / m_{B} \approx-0.024 \mathrm{fm}, \\
& \tilde{D}_{\frac{1}{m}}^{\prime} \approx-\frac{1}{48}\left[(D+3 F)^{2}-3(D-F)^{2}\right] \approx-0.086 / m_{B} \approx-0.017 \mathrm{fm}
\end{aligned}
$$

where $m_{B}$ is the (centroid) baryon mass which we take $\sim 1 \mathrm{GeV}$. We have used here the tree-level fits $F=0.44$ and $D=0.81$. There will also be contributions that arise from degrees of freedom whose mass scale is higher than the chiral expansion scale $\Lambda_{\chi} \sim 1 \mathrm{GeV}$. These can appear as a counter-term contribution and cannot be calculated in the scheme. They should be determined from experiments. In some cases such as in $\pi \pi$ scattering, those constants can be saturated by resonances (for instance, the constants $L_{i}$ in the Lagrangian of $O\left(Q^{4}\right)$ in the pion sector are dominated by the vector mesons $\rho, a_{1}$ etc.) but here while this is plausible, we have not succeeded in finding such a simple mechanism.

\section{On-Shell Constraints}

What can we say about the constants $\tilde{D}$ and $\tilde{D}^{\prime}$ from experiments?

For the purpose of determining these constants from experiments, it is simpler to look at $K^{+} N$ scattering. The $K^{-} N$ scattering is somewhat more delicate because of the resonance $\Lambda(1405)$. It has been suggested [6, 7, 8] that the presently available data on $K^{ \pm}$ nucleon and $K^{ \pm}$-nuclear scattering indicate that the bulk of the data can be understood reasonably well by the leading $\nu=1$ term (10) with the $\nu=2$ terms effectively suppressed. Let us see what this means in the framework of chiral perturbation theory. At present the data on threshold $K^{+} N$ scattering, particularly the isoscalar amplitudes, are not good enough to allow a precise determination of the constants. We can nonetheless make a simple analysis which is still meaningful as described below.

From the effective Lagrangian (10) and (11), we can immediately write down the expressions for scattering lengths

$$
\begin{aligned}
& a_{I=1}^{K N}=\frac{1}{4 \pi f^{2}\left(1+m_{K} / m_{B}\right)}\left(-m_{K}+\Sigma_{K N}+C+\left(\tilde{D}+\tilde{D}^{\prime}\right) m_{K}^{2}\right), \\
& a_{I=0}^{K N}=\frac{1}{4 \pi f^{2}\left(1+m_{K} / m_{B}\right)}\left(\Sigma_{K N}-3 C+\left(\tilde{D}-3 \tilde{D}^{\prime}\right) m_{K}^{2}\right) .
\end{aligned}
$$

where $a_{I=0,1}^{K N}$ are the S-wave scattering lengths for $K^{+} N$ scattering in isospin $I=0,1$. Given experimental values for $a_{I=0,1}^{K N}$ and knowing $\Sigma_{K N}$, these equations could determine the constants $\tilde{D}$ and $\tilde{D}^{\prime}$. 
Scattering lengths have recently been obtained from a rather complete analysis by Barnes and Swanson [15]. They find

$$
\begin{aligned}
& a_{I=1}^{K N} \approx-0.31 \mathrm{fm} \\
& a_{I=0}^{K N} \approx-0.09 \mathrm{fm} .
\end{aligned}
$$

Whereas $a_{I=1}^{K N}$ is reliably determined, $a_{I=0}^{K N}$ is highly uncertain: It can differ between 0 and $-0.2 \mathrm{fm}$ 16.

As long as $a_{I=0}^{K N}$ is small in magnitude compared with $a_{I=1}^{K N}$, the nuclear scattering of kaons should be insensitive to $a_{I=0}^{K N}$. This suggests a procedure which minimizes the dependence on the precise value of $a_{I=0}^{K N}$. The isospin averaged amplitude

$$
\bar{a}=\frac{3}{4} a_{I=1}^{K N}+\frac{1}{4} a_{I=0}^{K N}
$$

will occur in kaonic interactions in nuclear matter. In the case of neutron star matter, another combination occurs, but we will show that this latter case is insensitive to how we handle the on-shell kaon scattering. From (19), we find the empirical value

$$
\bar{a}_{e m p} \approx-0.255 \mathrm{fm}
$$

which is rather insensitive to the precise value of $a_{I=0}^{K N}$ within the given limits.

Müller-Groeling et al [8] have analyzed $\bar{K} N$ scattering in the framework of boson exchange. They employ vector mesons with roughly the same $S U(3)$ coupling coefficients as are implicitly incorporated in eq.(10). Whereas the scalar attraction between kaon and nucleon is built into the chiral Lagrangian (11) through the $\Sigma_{K N}$ term, Müller-Groeling et al [8] include an exchange of an explicit scalar particle with

$$
g_{\sigma \mathrm{NN}} g_{\sigma \mathrm{KK}} / 4 \pi \approx 0.9
$$

and $m_{\sigma} \approx 600 \mathrm{MeV}$. Using eqs. (30) and (31) of Brown, Koch and Rho [17], one can convert our sigma-term attraction into an effective $\sigma$-exchange by

$$
\left(\frac{g_{\sigma \mathrm{NN}} g_{\sigma \mathrm{KK}}}{4 \pi}\right)_{e f f}=\frac{1}{4 \pi} \frac{m_{\sigma}^{2} \Sigma_{K N}}{2 f^{2} m_{K}} \approx 0.93
$$

where we have used $\Sigma_{K N} \approx 2 m_{\pi}$ and $f=f_{\pi} \approx 93 \mathrm{MeV}$ in the conversion. The agreement between the Müller-Groeling et al value and ours is quite satisfactory. We thus see that

\footnotetext{
${ }^{4} \mathrm{~T}$. Barnes, private communication.
} 
there is evidence for attractive scalar interaction in the $\bar{K} N$ scattering consistent with $\Sigma_{K N} \approx 2 m_{\pi}$. p

To see the role of the constants $\tilde{D}$ and $\tilde{D}^{\prime}$, let us first set them equal to zero. Then from (17) and (18), we would predict (for $\Sigma_{K N} \approx 2 m_{\pi}$ )

$$
\begin{aligned}
a_{I=1}^{K N} & \approx-0.30 \mathrm{fm} \\
a_{I=0}^{K N} & =\frac{\Sigma_{K N}-3 C}{4 \pi f^{2}\left(1+m_{K} / m_{B}\right)} \approx 0.46 \mathrm{fm} .
\end{aligned}
$$

We have made clear in (25) that in the absence of the $\tilde{D}$ and $\tilde{D}^{\prime}$ corrections, the $a_{I=0}^{K N}$ is given mainly by the explicit chiral symmetry breaking. For this amplitude, the vector meson amplitude is zero. Equations (24) and (25) lead to $\bar{a}_{t h} \approx-0.11 \mathrm{fm}$ which is too small compared with the empirical value (21). Including the baryon-antibaryon pair contribution (16) brings $a_{I=0}^{K N}$ down to $0.40 \mathrm{fm}$, increasing $\bar{a}_{t h}$ to $\bar{a}_{t h} \approx-0.15 \mathrm{fm}$. Now if in addition to the pair contributions, one decreases the value of $\Sigma_{K N}$ by $30 \%$, then one gets $\bar{a} \approx-0.255 \mathrm{fm}$. Therefore it is not difficult to fit the amplitude $\bar{a}$ within the uncertainty of the parameters of the chiral Lagrangian. However it will then be difficult to understand in this way the scattering lengths $a_{I=0}^{K N}$ and $a_{I=1}^{K N}$ separately, even within the wide range of allowed values in $a_{I=0}^{K N}$.

Suppose we ignore the $\tilde{D}$ and $\tilde{D}^{\prime}$ terms but account for higher-order effects in the constants of the chiral Lagrangian. The most prominent quantity is $f$. Now at one loop (i.e., at $\nu=3$ ), the kaon field will scale with the kaon decay constant $f_{K}$, so $f$ should be replaced by $f_{K}$ in the sigma term $\sim \Sigma_{K N} / f^{2}$. On the other hand, the $\nu=1$ term (10) involves vector-meson exchange and requires that $f$ be identified with $f_{\pi}$, the pion decay constant. Calculation of other $\nu=3$ contributions has not yet been carried out, so it is difficult to quantify our argument but the use of $f_{K}$ instead of $f_{\pi}$ in the explicit symmetry breaking - which is equivalent to using $\left(\Sigma_{K N}\right)_{e f f} \approx 0.69 \Sigma_{K N}$ - generally moves our theoretical values towards the central values of the empirical ones. In fact a somewhat lower value

$$
\left(\Sigma_{K N}\right)_{e f f} \approx 0.6 \Sigma_{K N}
$$

would lead to an agreement between $\bar{a}_{t h}$ and $\bar{a}_{e m p}$.

\footnotetext{
${ }^{5}$ The hypothetical higher-mass scalar repulsion introduced by Büttgen et al [8] in the $K^{+} N$ channel is probably simulating the so-called "counter-term" contribution to $\tilde{D}$ and $\tilde{D}^{\prime}$ in the chiral Lagrangian discussed below and may be understood in the chiral bag language as a "van der Waals' repulsion" discussed by Vento et al [18].
} 
Let us now see what values of $\tilde{D}$ and $\tilde{D}^{\prime}$ are required to reproduce the "empirical" results (19). From (17) and (18), we find

$$
\begin{aligned}
\tilde{D} & \approx 0.33 / m_{K}-\Sigma_{K N} / m_{K}^{2}, \\
\tilde{D}^{\prime} & \approx 0.16 / m_{K}-C / m_{K}^{2} .
\end{aligned}
$$

For $\Sigma_{K N} \approx 2 m_{\pi}$, we have $\tilde{D} \approx-0.47 / m_{B}$ with $m_{B} \approx 2 m_{K}$. The constant $\tilde{D}^{\prime}$ is $\approx 0.46 / m_{B}$ independently of the value of the sigma term. (If one takes $a_{I=0}^{K N}=0$, one gets $\tilde{D} \approx$ $-0.41 / m_{B}$ and $\tilde{D}^{\prime} \approx 0.38 / m_{B}$ while for $a_{I=0}^{K N}=-0.2 \mathrm{fm}$, one finds $\tilde{D} \approx-0.57 / m_{B}$ and $\tilde{D}^{\prime} \approx 0.54 / m_{B}$.) Comparing with $(\sqrt{16})$, one sees that the pair term accounts for only a small part of the constants $\tilde{D}$ and $\tilde{D}^{\prime}$, the latter disagreeing even in sign. The conclusion then is that there must be additional corrections to the scattering lengths at order $\nu \geq 2$. These corrections can be of two different classes: One class would be of the $\nu=2$ terms coming from higher energy sector that is integrated out and is not saturated by the exchange of single resonances and the other would be loop corrections. As for the first class, we have no clue as to its mechanism. All we can do is to extract it from experiments. As for the second, since, as mentioned, there are no loop corrections to the $\tilde{D}$ and $\tilde{D}^{\prime}$ terms, they must enter with $\nu \geq 3$, contributing effectively to the scattering lengths as do $\tilde{D}$ and $\tilde{D}^{\prime}$ terms. Such calculations are in progress and will be reported elsewhere.

That loops could be important either in $\pi N$ scattering 9 or in $K N$ scattering or in both is pretty much obvious. In $\pi N$ scattering, our chiral Lagrangian (5) gives, at the $\nu=2$ order, the isoscalar $\pi N$ scattering length

$$
a_{\pi N}^{+} \equiv \frac{1}{2}\left(a^{\pi^{+} p}+a^{\pi^{+} n}\right)=\left[4 \pi f^{2}\left(1+m_{\pi} / m_{B}\right)\right]^{-1}\left(2 \tilde{D}_{\pi_{\mathrm{N}}} m_{\pi}^{2}+\Sigma_{\pi N}\right)
$$

where $\Sigma_{\pi N}$ is the $\pi N$ sigma term $\approx 45 \mathrm{MeV}$ and

$$
\tilde{D}_{\pi \mathrm{N}}=\frac{1}{4}\left(d_{1}+d_{2}\right)+\frac{1}{2}\left(d_{5}+d_{6}\right) .
$$

Now if one takes the empirical value of $a_{\pi N}^{+}$[19], $a_{\pi N}^{+}=-0.01 m_{\pi}^{-1}$, then one must have

$$
\tilde{D}_{\pi \mathrm{N}} \approx-0.27 \mathrm{fm}
$$

It should be noted, however, that the empirical value of $a_{\pi N}^{+}$can be reproduced by just taking the pair contribution alone (which is just a part of the $\tilde{D}$ term in the Lagrangian), or, stated differently, by the pseudovector pole contribution [19], or equivalently by the contribution

\footnotetext{
${ }^{6}$ This possibility was discussed in a somewhat different language by Delorme et al [19] and also by K. Kubodera and H. Yabu (private communication).
} 
of nucleon recoil [20]. This means in our scheme that loop contributions must be present such as to substantially cancel the contribution of $\Sigma_{\pi N}$ to the scattering length [19]. This could be explained by a mechanism that enhances the pair contribution to $\pi N$ scattering by order $\sim 2 m_{N} / m_{\pi} \rrbracket$.

In what follows, we will simply use the empirical values (27) and (28) effectively parametrizing $\nu \geq 2$ effects quadratic in kaon frequency. Later we will find that $K^{-}$condensation is little affected by these $\nu \geq 2$ effects. The reason can be seen from (11). Since the $\tilde{D}$ and $\tilde{D}^{\prime}$ multiply $\partial_{t} K \partial_{t} \bar{K}$, these corrections scale with density as $\omega_{\mathrm{K}}^{2} / m_{\mathrm{K}}^{2}$, where $\omega_{\mathrm{K}}$ is the kaon frequency. The loop corrections will also scale in the same way or what is more likely, even faster. The $\omega_{\mathrm{K}}$ decreases with increasing density, both because the kaon experiences the attractive scalar field of (23) and because of the attractive vector field from $\omega$-meson exchange with the nucleon described by the first term of (10). Thus the terms prefixed by $\tilde{D}$ and $\tilde{D}^{\prime}$ on the right-hand side of eq.(11) (and loop corrections absorbed in them) decrease compared with the first term with the coefficient $\Sigma_{K N} / f^{2}$. Consequently although the influence of the $\Sigma_{K N} / f^{2}$ term on the scattering lengths may be cut by $\sim 40$ $\%$ by the corrections, once the densities necessary for kaon condensation are reached, the influence of the correction terms will be greatly diminished.

In relativistic heavy-ion collisions, in which there is not enough time for strangeness violation, $\bar{K} K$ must be created [22]. Interactions of kaons and nucleons via $\omega$-meson exchange have opposite signs for $K$ and $\bar{K}$, so that they average out. Interactions via $\rho$-meson exchange go out for isospin-symmetric matter. The baryon-antibaryon pair term and loop corrections do not decrease with increasing density compared with $\Sigma_{K N} / f^{2}$ term, so that the corrections may become important. This issue would require a more careful treatment of higher chiral corrections.

\section{In-Medium Scaling}

So far we have been considering S-wave kaon interactions in free space. We would now like to take the chiral Lagrangian so defined and apply it to kaon interactions in nuclear matter. For this we have to account for the effect of the medium on chiral symmetry and other symmetries of QCD incorporated into the effective chiral Lagrangian. As argued by

\footnotetext{
${ }^{7}$ We conjecture that this enhancement may be understood by a mechanism analogous to the NambuJona-Lasinio picture of the pion in terms of $N \bar{N}$ bubbles. In effective field theories such as ours, such a phenomenon could occur - as in BCS theory - through a collective mechanism which turns "irrelevant" terms (of higher chiral order) into "marginal" terms. See Polchinski 21] on a discussion of this matter in the framework of effective field theories.
} 
Brown and Rho [9], the most economical way to implement chiral symmetry and trace anomaly of QCD - which are the most important properties of QCD at low energy - in nuclear medium is to endow to the in-medium effective chiral Lagrangian with the basic parameters of the theory that scale as a function of density. Arguments based on symmetries of QCD predict a universal scaling (valid at the mean field with the Lagrangian) of the quasiparticles relevant for the process in question [9]:

$$
\frac{m_{B}^{*}}{m_{B}} \approx \frac{m_{M}^{*}}{m_{M}} \approx \frac{f^{*}}{f}
$$

where the subscripts $B$ and $M$ stand, respectively, for baryons and mesons in the $S U(2)$ sector (except for Goldstone bosons) and the star denotes in-medium quantities at a density $\rho \neq 0$. The unstarred quantities denote free-space quantities. At the mean-field level, the constant $g_{A}$ remains unscaled [9]. There is by now rather strong evidence that the scaling (29) is valid in nuclei [24]. The consequence of this in-medium scaling on the effective Lagrangian (10) and (11) is simply that we replace $f$ by $f^{*}$. In medium, one is effectively including one-loop terms as mentioned above.

As alluded above, to one-loop order in free space, the kaon decay constant $f_{K}$ receives $O\left(Q^{2}\right)$ corrections relative to the pion decay constant $f_{\pi}$. So working to that level in medium, $f_{K}$ should be distinguished from $f_{\pi}$ in the effective Lagrangian, not only in its magnitude but also in its behavior. This could be implemented with a Lagrangian consisting of Goldstone bosons and baryons only. One possible way is to write, following Gasser and Leutwyler [25], a symmetry breaking term that involves derivatives of $O\left(M \partial^{2}\right)$, where $M$ is the quark mass matrix, and then perform a one-loop ChPT with this Lagrangian. This is very similar to what one does in skyrmion physics except that here only tree terms are considered. In this paper we shall not pursue this procedure. We find it far more transparent when the light vector mesons $\rho, \omega$, etc are explicitly present. Now in medium, we have $f_{\pi} \rightarrow f_{\pi}^{*} \approx \Phi(\rho) f_{\pi}$ whereas model calculations (such as NJL) indicate that $f_{K}$ scales very little up to $\rho \approx \rho_{0}$, so we could assume $f_{K}^{*} \approx f_{K}$ up to nuclear matter. [ This may be somewhat too naive but it should be more reasonable than taking it to scale like $f_{\pi}^{*}$. The consequence of this argument is then that in medium $f$ in eq.(10) is replaced by $f_{\pi}^{*}$ and that in eq.(11) by $f_{K}$. The replacement in (10) can be understood best in the description with explicit vector mesons. As noted in [9], the gauge coupling of the vector

\footnotetext{
${ }^{8}$ While there are no reliable lattice calculations in dense matter, we know what happens to the strange quark condensate $\langle\bar{s} s\rangle_{0}$ in high temperature. The calculation by Kogut et al 23. shows that the strangequark condensate changes little as a function of temperature while the light-quark condensate does. This is consistent with our assumption.
} 
meson $g$ remains unscaled at the mean-field level, so the scaling of $f_{\pi}^{*}$ means the scaling of the vector meson mass $m_{V}^{*}$ by the KSRF relation $m_{V}^{*}=2 f_{\pi}^{* 2} g^{2}$. Thus the factor $1 / f^{* 2}$ just corresponds to $2 g^{2} / m_{V}^{*}$ for the vector propagator in the medium at zero momentum transfer. We will simply assume that neither $\Sigma_{K N}$ nor $\tilde{D}$ nor $\tilde{D}^{\prime}$ scales with density. In reality, there may be some scaling in both: the former because it is related to the condensate difference between the vacuum and the hadronic "bag"; the latter because at least part of it may come from the pair term which depends inversely on the centroid baryon mass $m_{B}$ which presumably scales. We expect however that this is a fine-tuning that cannot be done accurately, so we will not pursue it any further.

The remaining procedure for describing kaon condensation in neutron star matter is identical to what was done in [3, 迎, so we will be very brief. Details are found in refs. [3, 4, 26]. We simply mention that in medium, the Lagrangians (10) and (11) generate one-loop terms and that kaon condensation will be triggered by the presence of electrons.

\section{Equation of State with Kaon Condensation}

The equation of state (EOS) describing the state of matter containing kaon condensates can be evaluated at the mean field level using the Lagrangian (5) with (6) and (7), retaining all nonlinear meson interactions. For this, loop corrections may be quantitatively important. In contrast, evaluating the critical density is relatively simple as it is insensitive to nonlinearities and loop corrections. Here we give the results for critical density and composition of the condensed matter computed with a chiral Lagrangian consistent with the on-shell constraints to the chiral order $\nu=2$.

Before implementing the $\tilde{D}$ and $\tilde{D}^{\prime}$ corrections, let us recall the previous results of refs. [3, 4, 26] obtained in the leading chiral order, $\nu=1$ supplemented by a $K N$ sigma term. The critical density is given as the nucleon density at which the pole of the kaon propagator $D$ is equal to to the electron chemical potential in the absence of the condensate. The electron chemical potential is determined by the nuclear matter equation of state and the conditions of beta equilibrium and local charge neutrality. Equivalently,the critical density is the nucleon density at which the energy density is lowered by the introduction of kaon condensate. In Table 1 9, we quote the results for the critical density without BR scaling

\footnotetext{
${ }^{9}$ With $a_{1} m_{s}=-67 \mathrm{MeV}$ and $a_{2} m_{s}=125 \mathrm{MeV}$ determined by Gell-Mann-Okubo mass formulas, the parameter $a_{3} m_{s}=-134,-222,-310 \mathrm{MeV}$ corresponds respectively to $\Sigma_{K N}=1.3 m_{\pi}, 2.5 m_{\pi}, 3.8 m_{\pi}$. The case $a_{3} m_{s}=-134 \mathrm{MeV}$ corresponds to $\langle\bar{s} s\rangle_{N} /\langle\bar{u} u\rangle_{N} \approx 0.07$. This represents the least favorable condition for kaon condensation and will be used for illustrating the equation of state we predict. Note that the difference from eq.(2) is related to the well-known $\Sigma_{\pi N}$ problem in the tree order of chiral Lagrangians, which is resolved by going to higher orders in chiral perturbation theory. In the present problem, it is best
} 
as given in refs. [4, 26], which are essentially those of [3] but with the inclusion of muons in beta equilibrium with the neutron star matter. Here as well as in what follows, we use the potential contribution to the symmetry energy $F(u)=u$ [3, 26, 27].

\section{TABLE 1}

The critical densities without $\tilde{D}$ and $\tilde{D}^{\prime}$ terms and without BR scaling. The parameters held fixed are: $a_{1} m_{s}=-67 \mathrm{MeV}, a_{2} m_{s}=125 \mathrm{MeV}, f_{K} \approx f_{\pi} \approx 93$ $\mathrm{MeV}$.

\begin{tabular}{lrc}
\hline \hline$F(u)$ & $a_{3} m_{s}(\mathrm{MeV})$ & $u_{c}$ \\
\hline \multirow{3}{*}{$u$} & -134 & 4.11 \\
& -222 & 3.04 \\
& -310 & 2.39 \\
\hline \hline
\end{tabular}

Next we incorporate $\tilde{D}$ and $\tilde{D}^{\prime}$ terms so as to be consistent (at the order $\nu=2$ ) with the $K^{+} N$ scattering lengths. In what follows, we will simply take (27) and (28) valid to order $\nu=2$. A more sophisticated analysis would require more accurate $K^{+} N$ data and loop corrections. The inverse propagator is

$$
\begin{aligned}
D^{-1}(\omega) & =\omega^{2}-m_{K}^{2}-\Pi(\omega) \\
& =\left(1+\left[\frac{\tilde{D}}{f^{2}}+(2 x-1) \frac{\tilde{D}^{\prime}}{f^{2}}\right] u \rho_{0}\right) \omega^{2}-m_{K}^{2}+u \rho_{0} \frac{1+x}{2 f^{2}} \omega+\frac{u \rho_{0}}{f^{2}}\left(\Sigma^{K N}+(2 x-1) C\right) .
\end{aligned}
$$

\section{TABLE 2}

The critical densities in ChPT with $\nu=2$ terms without $\left(u_{c}^{n s}\right)$ and with $\left(u_{c}^{s 1, s 2}\right)$ BR scaling. For parameter values, see Table 1.

\begin{tabular}{lrccc}
\hline \hline$F(u)$ & $a_{3} m_{s}(\mathrm{MeV})$ & $u_{c}^{n s}$ & $u_{c}^{s 1}$ & $u_{c}^{s 2}$ \\
\hline \multirow{4}{*}{$u$} & -134 & 4.20 & 2.84 & 3.15 \\
& -222 & 3.27 & 2.49 & 2.69 \\
& -310 & 2.60 & 2.16 & 2.39 \\
\hline \hline
\end{tabular}

to consider the numerical value of " $\Sigma_{K N}$ " as a parameter. 
The critical density computed with eq.(30) without BR scaling is given in Table 1, denoted as $u_{c}^{n s}$. To implement the BR scaling in eq.(30), we replace $f_{\pi} \rightarrow f_{\pi}^{*}$ and $f_{K} \rightarrow f_{K} \approx f_{\pi}$ in the manner prescribed above. (We are disregarding the $O\left(Q^{2}\right)$ correction to $f_{K}$ in our numerical estimates.) As argued above, this corresponds to scaling the vector interaction only. Now if we take the scaling to be $f_{\pi}^{*}=f_{\pi} /(1+0.25 u)$, we get the critical density labeled $u_{c}^{s 1}$ in Table 2. The results are only slightly changed if one uses a slower scaling, say, $f_{\pi}^{*}=f_{\pi} /(1+0.16 u)$. They are given in Table 2 as $u_{c}^{s 2}$.

The composition of the condensed matter is illustrated in Tables 3 and 4 . In Table 3 are given the results obtained without BR scaling and in Table 4 those with BR scaling with $f_{\pi}^{*} / f_{\pi} \approx(1+0.25 u)^{-1}$. We have shown only the results with the small $\Sigma_{K N} \approx 1.3 m_{\pi}$ (or $\left.a_{3} m_{s}=-134 \mathrm{MeV}\right)$ to illustrate the robustness of the prediction. The quantities reported in the Tables are: The chiral angle $\theta$, the energy density gain $\Delta \epsilon$ (in $\mathrm{MeV} / \mathrm{fm}^{3}$ ), the chemical potential $\mu$ (in $\mathrm{MeV}$ ), the proton fraction $x$, the kaon fraction $x_{K}=\rho_{K} / \rho$, the electron fraction $x_{e}=\rho_{e} / \rho$ and the muon fraction $x_{\mu}=\rho_{\mu} / \rho$ as a function of matter density $u=\rho / \rho_{0}$.

\section{TABLE 3}

"Nuclear star" composition without BR scaling for $\Sigma_{K N} \approx 1.3 m_{\pi}\left(a_{3} m_{s} \approx-134\right.$ $\mathrm{MeV})$.

\begin{tabular}{rrrrrrrr}
\hline \hline $\mathrm{u}$ & $\theta$ & $\Delta \epsilon$ & $\mu$ & $x$ & $x_{K}$ & $x_{e}$ & $x_{\mu}$ \\
\hline 4.20 & 0.4 & 0.0 & 256.7 & 0.195 & 0.000 & 0.111 & 0.085 \\
4.70 & 31.6 & -3.0 & 223.9 & 0.291 & 0.180 & 0.066 & 0.046 \\
5.20 & 43.6 & -11.2 & 191.3 & 0.360 & 0.301 & 0.037 & 0.022 \\
5.70 & 51.7 & -23.7 & 159.9 & 0.409 & 0.380 & 0.020 & 0.009 \\
6.20 & 57.4 & -39.3 & 130.8 & 0.444 & 0.431 & 0.010 & 0.002 \\
6.70 & 61.5 & -57.4 & 104.5 & 0.468 & 0.464 & 0.005 & 0.000 \\
7.20 & 64.5 & -77.1 & 81.1 & 0.486 & 0.484 & 0.002 & 0.000 \\
7.70 & 66.8 & -98.3 & 60.4 & 0.499 & 0.499 & 0.001 & 0.000 \\
8.20 & 68.6 & -120.5 & 42.0 & 0.509 & 0.509 & 0.000 & 0.000 \\
8.70 & 70.0 & -143.5 & 25.8 & 0.517 & 0.517 & 0.000 & 0.000 \\
9.20 & 71.1 & -167.3 & 11.3 & 0.522 & 0.522 & 0.000 & 0.000 \\
9.70 & 72.0 & -191.6 & -1.7 & 0.526 & 0.526 & 0.000 & 0.000 \\
10.20 & 72.7 & -216.5 & -13.4 & 0.530 & 0.530 & 0.000 & 0.000 \\
\hline \hline
\end{tabular}


Some remarkable features in the results are as follows.

- The critical densities are robust with respect to the parameters of the effective Lagrangian. The on-shell constraints bring only small modification. The in-medium scaling reduces the critical density from $u_{c} \sim 4$ to $u_{c} \sim 3$.

- With the in-medium scaling, the critical density is fairly insensitive to the numerical value of $\Sigma_{K N}$.

- Once kaon condensate sets in, the initially dense neutron matter turns quickly to nuclear matter, with the proton fraction $x$ being already quite substantial just above the critical density. This shows that with kaon condensates, a compact star is more likely a "nuclear star" rather than neutron star of the standard scenario.

- The kaon fraction becomes equal to the proton fraction slightly above the critical density; the proton charge is almost entirely balanced by the $K^{-}$charge.

\section{TABLE 4}

"Nuclear star" composition with BR scaling with $f_{\pi}^{*} / f_{\pi}=(1+0.25 u)^{-1}$ and $\Sigma_{K N} \approx 1.3 m_{\pi}\left(a_{3} m_{s} \approx-134 \mathrm{MeV}\right.$. $)$

\begin{tabular}{rrrrrrrr}
\hline \hline $\mathrm{u}$ & $\theta$ & $\Delta \epsilon$ & $\mu$ & $x$ & $x_{K}$ & $x_{e}$ & $x_{\mu}$ \\
\hline 2.84 & 0.0 & 0.0 & 209.6 & 0.147 & 0.000 & 0.089 & 0.058 \\
3.34 & 28.0 & -7.0 & 134.5 & 0.356 & 0.331 & 0.020 & 0.005 \\
3.84 & 30.2 & -19.5 & 92.8 & 0.424 & 0.419 & 0.006 & 0.000 \\
4.34 & 29.9 & -33.3 & 66.0 & 0.457 & 0.455 & 0.002 & 0.000 \\
4.84 & 29.0 & -47.5 & 47.6 & 0.475 & 0.474 & 0.001 & 0.000 \\
5.34 & 27.8 & -61.7 & 34.6 & 0.485 & 0.485 & 0.000 & 0.000 \\
5.84 & 26.6 & -76.1 & 25.3 & 0.491 & 0.491 & 0.000 & 0.000 \\
6.34 & 25.5 & -90.5 & 18.4 & 0.495 & 0.495 & 0.000 & 0.000 \\
6.84 & 24.4 & -105.0 & 13.2 & 0.498 & 0.498 & 0.000 & 0.000 \\
7.34 & 23.3 & -119.6 & 9.3 & 0.499 & 0.499 & 0.000 & 0.000 \\
7.84 & 22.4 & -134.4 & 6.3 & 0.501 & 0.501 & 0.000 & 0.000 \\
8.34 & 21.5 & -149.3 & 4.0 & 0.501 & 0.501 & 0.000 & 0.000 \\
8.84 & 20.6 & -164.5 & 2.2 & 0.502 & 0.502 & 0.000 & 0.000 \\
9.34 & 19.9 & -179.9 & 0.8 & 0.502 & 0.502 & 0.000 & 0.000 \\
9.84 & 19.2 & -195.4 & -0.2 & 0.502 & 0.502 & 0.000 & 0.000 \\
\hline \hline
\end{tabular}




\section{Conclusion: Astrophysical Consequences}

Our results show that the critical density is remarkably robust. It comes out to be $u_{c} \sim(3-4)$ quite independently of the constraints from kaon-nuclear interactions. What is also noteworthy is that once the BR scaling is implemented, the dependence on the strangeness content of the proton is considerably weaker. Even when one assumes that $\langle N|\bar{s} s| N\rangle=0$ which is certainly unreasonably conservative, the critical density is $u_{c}<3$. This robust nature of the condensation leads to some striking consequences on stellar collapse.

Kaon condensation plays an important role in the collapse of large stars, once central densities exceed the critical density $u_{c}$. Brown et al [3] showed that electrons changed into $K^{-}$-mesons and neutrinos in a fraction of a second, the neutrinos being trapped for a longer time (of $\sim 10$ seconds). Since a large fraction of protons are present in kaon condensation, nuclear matter rather than neutron matter - formed in the conventional scenario - is reached and this has the effect of substantially softening the dense matter equation of state (EOS) because of lower symmetry energy of nuclear matter. Because of the greater binding energy, more energy will be emitted in neutrinos [26].

Effects of kaon condensation on the supernova explosion mechanism remain to be explored. However effects of kaon condensation on the structure of neutron stars and on some aspects of the explosion have been worked out by Thorsson et al 26.

Because kaon condensation softens the nuclear EOS at high densities, it substantially diminishes the maximum mass $M_{\max }$ for neutron stars. Brown [28] found, with reasonable assumption about the compression modulus of nuclear matter that

$$
M_{\text {max }} \approx 1.5 M_{\odot} .
$$

This has major implications for the formation of black holes in stellar collapse [5]. Stars in the range of $\sim 18$ to $30 M_{\odot}$ can first explode, returning matter to the galaxy and then go into low-mass black holes of mass $M_{\mathrm{BH}} \sim 1.5 M_{\odot}$. Upon collapse, stars heavier than $\sim 30 M_{\odot}$ drop directly into black holes without nucleosynthesis. These black holes are heavier, of mass $M_{\mathrm{BH}} \geq 10 M_{\odot}$. Brown and Bethe [5] estimate that $\sim 10^{9}$ black holes have been formed in the above way in the galaxy.

\section{Acknowledgments}

We are grateful for useful correspondence from K. Kubodera, M. Prakash and H. Yabu on related problems and for helpful comments from W. Weise. One of us (CHL) would like to acknowledge valuable discussions with $\mathrm{H}$. Jung with whom further work is in progress. 


\section{Appendix}

Here we sketch briefly the derivation of the $1 / m_{B}$ corrections. Consider the lowest order Lagrangian with baryon fields,

$$
\mathcal{L}=\operatorname{Tr} \bar{B}\left(i \not D-m(1-\not) B+\mathrm{F} \gamma^{\mu} \gamma_{5}\left[A_{\mu}, B\right]+\mathrm{D} \gamma^{\mu} \gamma_{5}\left\{A_{\mu}, B\right\}\right)
$$

Rewriting the fields as $B=\frac{1}{\sqrt{2}} B^{a} \lambda^{a}, V_{\mu}=V_{\mu}^{a} \lambda^{a}$ and $A_{\mu}=A_{\mu}^{a} \lambda^{a}$, where $\lambda^{a}, a=1,8$ are the Gell-Mann matrices, the Lagrangian can be written

$$
\mathcal{L}=\bar{B}^{a} i \not D^{a c} B^{c}-m \bar{B}^{a} \delta^{a c}(1-\psi) B^{c}+\bar{B}^{a} \gamma^{\mu} \gamma_{5} \mathcal{A}_{\mu}^{a c} B^{c}
$$

Here

$$
\begin{aligned}
i \not D^{a c} & =i \delta^{a c} \not \partial+i\left(2 i f^{a b c}\right) Y^{b} \\
\mathcal{A}_{\mu}^{a c} & =\mathrm{F}\left(2 i f^{a b c}\right) A_{\mu}^{b}+\mathrm{D}\left(2 d^{a b c}\right) A_{\mu}^{b}
\end{aligned}
$$

and $f^{a b c}, d^{a b c}$ are $S U(3)$ structure constants. The equation of motion for the heavy baryon field is

$$
\left(g^{a c}-m(1-\psi) \delta^{a c}\right) B^{c}=0
$$

with $g^{a c}=i \not D^{a c}+\gamma^{\mu} \gamma_{5} \mathcal{A}_{\mu}^{a c}$. Now, decompose $B^{a}$ using $P_{+}=\frac{1}{2}(1+\psi)$ and $P_{-}=\frac{1}{2}(1-\psi)$,

$$
B^{a}=P_{+} B^{a}+P_{-} B^{a}=B_{+}^{a}+B_{-}^{a} .
$$

Applying $P_{-}$to eq. A.4 from the left, we have

$$
B_{-}^{c}=\frac{1}{2 m} P_{-} g^{c e} B_{+}^{e}+O\left(\frac{1}{m^{2}}\right) .
$$

Now applying $P_{+}$to eq.(A.4) and using eq.A.6), we get, modulo $O\left(1 / m^{2}\right)$,

$$
P_{+}\left(g^{a e}+\frac{1}{2 m} g^{a c} P_{-} g^{c e}\right) B_{+}^{e}=0 .
$$

Thus the Lagrangian containing $1 / m_{B}$ correction term is

$$
\mathcal{L}=\bar{B}_{+}^{a}\left(g^{a e}+\frac{1}{2 m} g^{a c} P_{-} g^{c e}\right) B_{+}^{e} .
$$

To write this explicitly, we note that the $O\left(A^{2}\right)$ terms are given by

$$
g^{a c} P_{-} g^{c e}=\gamma^{\mu} \gamma_{5} \mathcal{A}_{\mu}^{a c} P_{-} \gamma^{\nu} \gamma_{5} \mathcal{A}_{\nu}^{c e}=-\mathcal{A}^{a c} P_{+} \mathcal{A}^{c e}=-\left(v \cdot \mathcal{A}^{a c}\right)\left(v \cdot \mathcal{A}^{c e}\right)
$$


which leads to $\left(m=m_{B}\right)$

$$
\mathcal{L}_{1 / m} \longrightarrow-\frac{1}{2 m_{B}} \bar{B}^{a}\left(v \cdot \mathcal{A}^{a c}\right)\left(v \cdot \mathcal{A}^{c e}\right) B^{e} .
$$

From the identities

$$
\begin{aligned}
\left(2 i f^{a b c}\right)\left(2 i f^{c d e}\right) & =\frac{1}{2} \operatorname{Tr}\left(\lambda^{a}\left[\lambda^{b},\left[\lambda^{d}, \lambda^{e}\right]\right]\right), \\
\left(2 i f^{a b c}\right)\left(2 d^{c d e}\right) & =\frac{1}{2} \operatorname{Tr}\left(\lambda^{a}\left[\lambda^{b},\left\{\lambda^{d}, \lambda^{e}\right\}\right]\right), \\
\left(2 d^{a b c}\right)\left(2 i f^{c d e}\right) & =\frac{1}{2} \operatorname{Tr}\left(\lambda^{a}\left\{\lambda^{b},\left[\lambda^{d}, \lambda^{e}\right]\right\}\right), \\
\left(2 d^{a b c}\right)\left(2 d^{c d e}\right) & =\frac{1}{2} \operatorname{Tr}\left(\lambda^{a}\left\{\lambda^{b},\left\{\lambda^{d}, \lambda^{e}\right\}\right\}\right)-\frac{2}{3} \operatorname{Tr}\left(\lambda^{a} \lambda^{b}\right) \operatorname{Tr}\left(\lambda^{d} \lambda^{e}\right),
\end{aligned}
$$

we have

$$
\begin{aligned}
\bar{B}^{a}\left(v \cdot \mathcal{A}^{a c}\right)\left(v \cdot \mathcal{A}^{c e}\right) B^{e}= & \operatorname{Tr} \bar{B}\left(\mathrm{D}^{2}\{v \cdot A,\{v \cdot A, B\}\}+\mathrm{DF}\{v \cdot A,[v \cdot A, B]\}\right. \\
& \left.+\mathrm{F}^{2}[v \cdot A,[v \cdot A, B]]+\mathrm{FD}[v \cdot A,\{v \cdot A, B\}]\right) \\
& -\frac{4}{3} \mathrm{D}^{2} \operatorname{Tr}(\bar{B} v \cdot A) \operatorname{Tr}(v \cdot A B) \\
= & (\mathrm{D}+\mathrm{F})^{2} \operatorname{Tr} \bar{B}(v \cdot A)^{2} B+2\left(\mathrm{D}^{2}-\mathrm{F}^{2}\right) \operatorname{Tr} \bar{B}(v \cdot A) B(v \cdot A) \\
& +(\mathrm{D}-\mathrm{F})^{2} \operatorname{Tr} \bar{B} B(v \cdot A)^{2}-\frac{4}{3} \mathrm{D}^{2} \operatorname{Tr}(\bar{B} v \cdot A) \operatorname{Tr}(v \cdot A B) .
\end{aligned}
$$

For S-wave scattering,

$$
\begin{aligned}
\operatorname{Tr} \bar{B} A_{0}^{2} B & \longrightarrow \frac{1}{4 f^{2}}\left((\bar{N} N)\left(\partial_{t} \bar{K} \partial_{t} K\right)+(\bar{N} \vec{\tau} N) \cdot\left(\partial_{t} \bar{K} \vec{\tau} \partial_{t} K\right)\right) \\
\operatorname{Tr}\left(\bar{B} A_{0}\right) \operatorname{Tr}\left(A_{0} B\right) & \longrightarrow \frac{1}{4 f^{2}}\left((\bar{N} N)\left(\partial_{t} \bar{K} \partial_{t} K\right)+(\bar{N} \vec{\tau} N) \cdot\left(\partial_{t} \bar{K} \vec{\tau} \partial_{t} K\right)\right) \\
\operatorname{Tr} \bar{B} B A_{0}^{2} & \longrightarrow \frac{1}{2 f^{2}}(\bar{N} N)\left(\partial_{t} \bar{K} \partial_{t} K\right) \\
\operatorname{Tr} \bar{B} A_{0} B A_{0} & \longrightarrow 0
\end{aligned}
$$

The $1 / m_{B}$ corrections are therefore

$$
\mathcal{L}_{\frac{1}{m}}=\tilde{D} \frac{1}{f^{2}}\left(\bar{N} N \partial_{t} \bar{K} \partial_{t} K\right)+\tilde{D}^{\prime} \frac{1}{f^{2}}(\bar{N} \vec{\tau} N) \cdot\left(\partial_{t} \bar{K} \vec{\tau} \partial_{t} K\right)
$$

with

$$
\begin{aligned}
& \tilde{D}_{\frac{1}{m}}=-\frac{1}{48 m_{B}}\left((\mathrm{D}+3 \mathrm{~F})^{2}+9(\mathrm{D}-\mathrm{F})^{2}\right) \\
& \tilde{D}_{\frac{1}{m}}^{\prime}=-\frac{1}{48 m_{B}}\left((\mathrm{D}+3 \mathrm{~F})^{2}-3(\mathrm{D}-\mathrm{F})^{2}\right) .
\end{aligned}
$$




\section{References}

[1] D.B. Kaplan and A.E. Nelson, Phys. Lett. B175 (1986) 57

[2] H.D. Politzer and M.B. Wise, Phys. Lett. B273 (1991) 156

[3] G.E. Brown, K. Kubodera, M. Rho and V. Thorsson, Phys. Lett. B291 (1992) 355

[4] V. Thorsson, Ph.D. thesis, SUNY at Stony Brook (1992), unpublished

[5] G.E. Brown and H.A. Bethe, "A scenario for a large number of low mass black holes in the galaxy," CALTECH preprint, submitted to Astrophysical Journal (1993)

[6] See, e.g., W. Weise, Proceedings Int. Nucl. Phys. Conf., Wiesbaden, 27 July-1 August 1992; Nucl. Phys. A, to be published

[7] P.B. Siegel and W. Weise, Phys. Rev. C38 (1988) 221

[8] A. Müller-Groeling, K. Holinde and J. Speth, Nucl. Phys. A513 (1990) 557; R. Büttgen, K. Holinde, A. Müller-Groeling, J. Speth and P. Wyborny, Nucl. Phys. A506 (1990) 586

[9] G.E. Brown and M. Rho, Phys. Rev. Lett. 66, 2720 (1991)

[10] E. Jenkins and A. Manohar, Phys. Lett. B255 (1991) 558; B259 (1991) 353; E. Jenkins, Nucl. Phys. B368 (1991) 190

[11] S. Weinberg, Phys. Lett. B251 (1990) 288; Nucl. Phys. B363 (1991) 2

[12] T.-S. Park, D.-P. Min and M. Rho, "Chiral dynamics and heavy-fermion formalism in nuclei," submitted to Phys. Repts. (1993)

[13] M. Bando, T. Kugo and K. Yamawaki, Phys. Repts. 164 (1988) 217

[14] J. Gasser, M.E. Sainio and A. Švarc, Nucl. Phys. B307 (1988) 779

[15] T. Barnes and E.S. Swanson, MIT-CTP-2169/ORNL-CCIP-92-15 (December 1992)

[16] J.S. Hyslop, R.A. Arndt, L.D. Roper and R.L. Workman, Phys. Rev. D46 (1992) 961

[17] G.E. Brown, V. Koch and M. Rho, Nucl. Phys. A535 (1991) 701

[18] V. Vento, M. Rho and G.E. Brown, Phys. Lett. B103 (1981) 285 
[19] J. Delorme, M. Ericson and T.E.O. Ericson, Phys. Lett. B291 (1992) 379

[20] J. Schwinger, "Particles and Sources ", Gordon and Breach, New York (1969)

[21] J. Polchinski, "Effective field theory and the Fermi surface," 1992 TASI Lecture

[22] A.E. Nelson and D.B. Kaplan, Phys. Lett. B192 (1987) 193; C.M. Ko, Z.G. Wu, L.H. Xia and G.E. Brown, Phys. Rev. Lett. 66 (1991) 2577; G.E. Brown, C.M. Ko, Z.G. Wu and L.H. Xia, Phys. Rev. C43 (1991) 1881

[23] J.B. Kogut, D.K. Sinclair and K.C. Wang, Phys. Lett. 263 (1991) 101

[24] C. Adami and G.E. Brown, Phys. Repts., to appear

[25] J. Gasser and H. Leutwyler, Ann. Phys. (N.Y.) 158, (1984) 142; Nucl. Phys. B307 (1988) 763;(1991) 353

[26] V. Thorsson, M. Prakash and J.M. Lattimer, "Composition, structure and evolution of neutron stars with kaon condensates", NORDITA-93/29 N / SUNY-NTG-92-33

[27] M.Prakash, T. L. Ainsworth and J.M. Lattimer, Phys. Rev. Lett. 61 (1988) 2518

[28] G.E. Brown, Proc. First Symp. on Nucl. Phys. of the Universe, Oak Ridge, TN, September 24-26, 1992, to be published. 